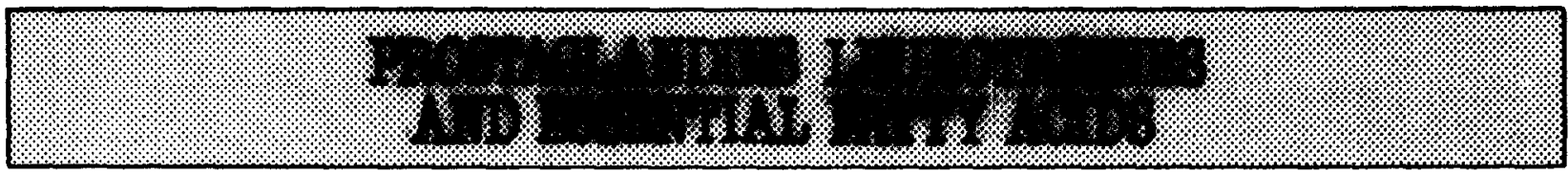

Prostaglandins Leukotrienes and Essential Fatty Acids (1991) 43, 55-59 (C) Longman Group UK Lid 1991

\title{
15-HETE is the Main Eicosanoid Present in Mucus of Ulcerative Proctocolitis
}

\author{
F. J. Zijlstra and J. H. P. Wilson*
}

Department of Pharmacology, Erasmus University, P.O. Box 1738, 3000 DR Rotterdam, The Netherlands and *Department of Gastroenterology, Academic Hospital Dijkzigt, Dr Molewaterplein 40, 3015 GD Rotterdam, The Netherlands (Reprint requests to FJZ)

$A B S T R A C T$. Prostaglandins, leukotrienes and mono-hydroxy acid products of arachidonic acid were measured in mucus of freshly recovered morning stools of a patient with an exacerbation of ulcerative proctocolitis. Eicosanoids in ether extracts were separated by high performance liquid chromatography and amounts determined by radioimmunoassay. Four hydroxy-eicosatetraenoic acids were detected, of which the most important one was identified as 15-hydroxy eicosatetraenoic acid ( $530 \mathrm{ng} / \mathrm{g}$ mucus). Leukotriene $\mathbf{B}_{4}$ was also present (21 ng/g mucus) and small amounts of immunoreactive leukotriene $C_{4}$ ( $<0.8 \mathrm{ng} / \mathrm{g}$ mucus). The prostaglandins 6-keto-PGF $1 \alpha$ and $\mathrm{PGE}_{2}$ and thromboxane $\mathrm{B}_{2}$ were found in amounts of $3.7,2.0$ and $9.2 \mathrm{ng} / \mathrm{g}$ mucus, respectively.

\section{INTRODUCTION}

It has been suggested that lipoxygenase products play a role in bowel disease and in particular in ulcerative colitis $(1,2)$. In inflammatory bowel disease, the synthesis of leukotriene $\mathrm{B}_{4}\left(\mathrm{LTB}_{4}\right)$ in colonic mucosa has been shown to be enhanced compared to normal tissue (3). Using equilibrium dialysis of the rectum Lauritsen et al found increased concentration of prostaglandin $E_{2}$ and $F_{2 \alpha}$ and thromboxane $B_{2}$ in rectal dialysates of patients with ulcerative colitis, Crohn's colitis and Clostridium difficile colitis, and substantially increased levels of leukotriene $B_{4}$ in patients with ulcerative colitis $(4,5)$. Effects of prednisolone and 5-aminosalicylic acid on these $\mathbf{L T B}_{4}$ levels were investigated as well as proven selective 5-lipoxygenase inhibitors (6). In these in vivo investigations a significant decrease of $\mathrm{LTB}_{4}$ was observed, however the clinical efficacy still must be shown in controlled trials (7).

In several animal models of inflammatory bowel disease effects of leukotriene synthesis inhibitors is investigated. Acute and chronic models have been developed in rats (8), rabbits (9) and mice (10).

Date received 6 October 1990

Date accepted 3 January 1991
Most studies were focussed on the synthesis or measurements of levels of $\mathrm{LTB}_{4}$ and $\mathrm{PGE}_{2}$. Recently more evidence for the involvement of other 5-lipoxygenase products, the sulfidopeptideleukotrienes, was found $(11,12)$. Studies in which the metabolism of exogenous arachidonic acid in colonic mucosa in inflammatory bowel disease was investigated, also showed a 3-5-fold increase of other eicosanoids, such as 12- and 15-HETE and HHT (3). It is well known that mono-HETEs act as mediators in mucus secretion (13) and have inflammatory effects in skin (14).

In the present study, lipoxygenase products were determined in intestinal mucus from an individual with mild ulcerative proctocolitis. Mucus secreted with stool was collected from fresh morning stools for 4 days, separated and extracted with diethylether. The eicosanoids were determined and identified by high performance liquid chromatography (HPLC) and radioimmunoassay (RIA). High levels of another lipoxygenase product, 15-HETE, were found in addition to $\mathrm{LTB}_{4}$.

\section{MATERIALS AND METHODS}

\section{Patient history}

The patient was a 35-year-old man. He started to lose blood with his stools when he was 15 . After 
2 years of complaints he was admitted to hospital when fiberoptic sigmoidoscopy revealed a friable mucosa, frank bleeding and some pseudopolyps. The histology was compatible with ulcerative colitis and a diagnosis of ulcerative proctocolitis was made. A remission was induced with prednisone and sulfasalazine. Several recurrences occurred which responded to prednisone and sulfasalazine. Ten years thereafter colonoscopy again revealed friable mucosa in the rectum and sigmoid with normal mucosa in the transverse and descending colon. Treatment with prednisone was again instituted after a rash developed on sulfasalazine. The steroids were gradually tapered off. Since then the patient has not been on drug therapy. A fiberoptic sigmoidoscopy showed a very mild proctocolitis which was confirmed by histology. At the time of the study he was feeling well, had no fever and produced a stool of normal consistence surrounded by mucus, once daily (15).

\section{Methods}

Morning stools were collected and mucus separated immediately. Extraction was performed with diethylether ( $5 \mathrm{ml}$ per gram mucus). Tritiated prostaglandins (PGs), leukotrienes (LTs) and mono-hydroxyeicosatetraenoic acids (HETEs) were added to measure recoveries. After centrifugation $(5 \mathrm{~min}$, $2800 \mathrm{xg}$ ) the supernatant was collected and evaporated under nitrogen at $40^{\circ} \mathrm{C}$. The extract was dissolved in $300 \mathrm{ml}$ of HPLC solvent system and filtered over an Acro C3A $0.45 \mathrm{~mm}$ filter (Gelman). The sample was kept in a plastic micro vial (Weichmann, Switzcrland). One hundred $\mathrm{ml}$ was injected into a ChromSep Nucleosil $5 \mathrm{C}_{18}$ column (Chrompack, Middelburg, The Netherlands) using a 1084B high performance liquid chromatograph of Hcwlctt Packard. The solvent system contained: tetrahydrofuran-methanol- $0.1 \%$ EDTA in wateracetic acid $(25: 30: 45: 0.1)$ adjusted to $\mathrm{pH} 5.5$ with ammonium hydroxide. The flow rate was $0.4 \mathrm{ml} / \mathrm{min}$, oven temperature $37^{\circ} \mathrm{C}$. Absorption was monitored as indicated in the Figure. Fractions of 1 per min were collected by a LKB Superrac. Twentyfive ml portions were used for RIA of LTs and HEs. PGs which coelute with LTC $_{4}$ were measured by RIA of 6-keto-prostaglandin $\mathrm{F}_{1 \alpha}$ (6-keto-PGF $\mathrm{F}_{1 \alpha}$ ), thromboxane $\mathrm{B}_{2}\left(\mathrm{TxB}_{2}\right)$ and prostaglandin $\mathrm{E}_{2}$ $\left(\mathrm{PGE}_{2}\right)$, for which purposes $25 \mathrm{ml}$ of the fractions 4-8 were used. The method was described earlier, in detail (16).

\section{Chemicals}

Synthetic LTs were generous gifts of Dr. J. Rokach (Merck Frosst Labs, Canada), mono-HETEs were obtained from Seragen (Boston, MA, USA) as well
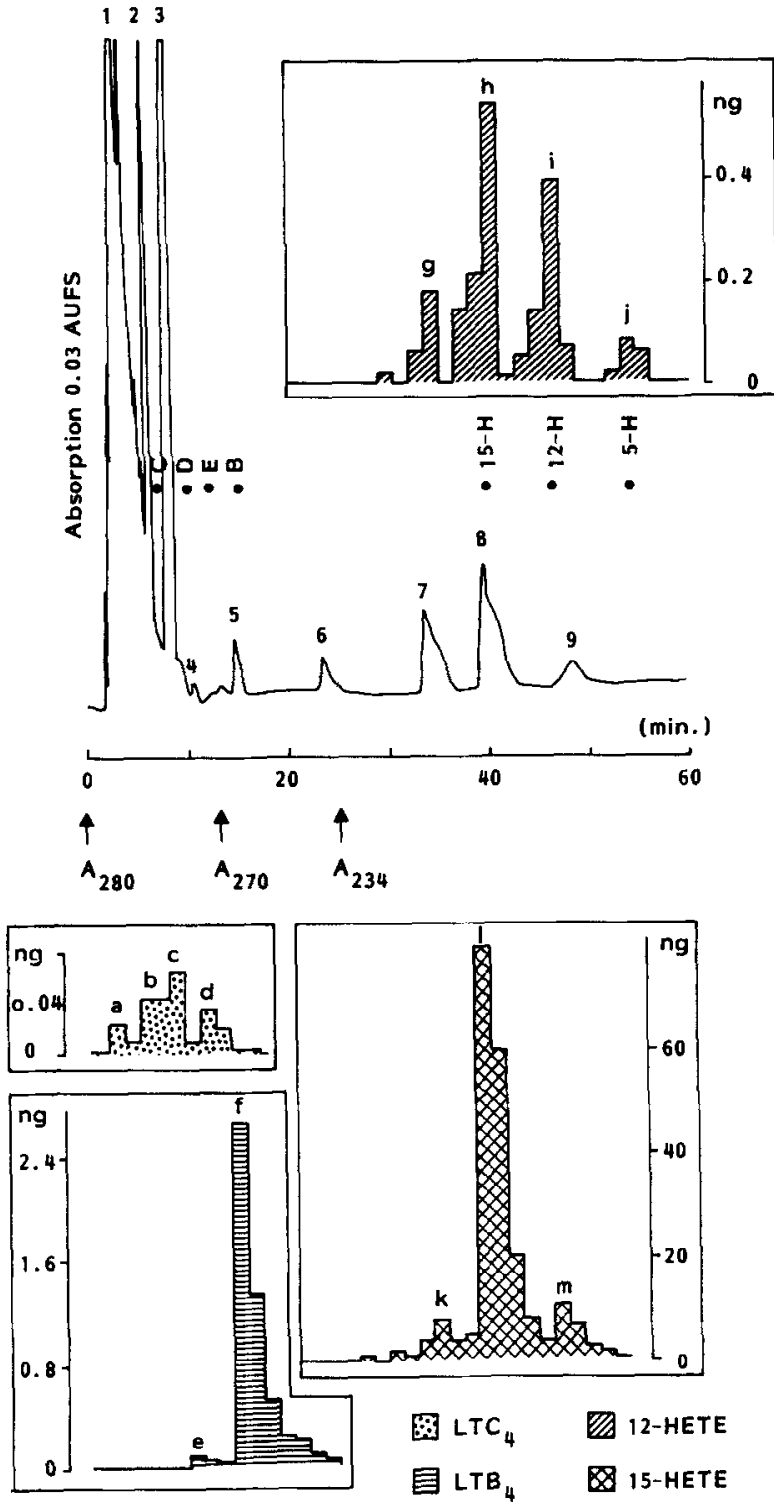

Fig. RP-HPLC separation of PGs, LTs and HEs in an extracted mucus sample of a patient with ulcerative procto colitis. A ChromSep Nucleosil $5 \mathrm{C}_{18}$ column $(200 \times 3.0 \mathrm{~mm})$ was used. Mobile phase: tetrahydrofuran-methanol- $0.1 \%$ EDTA in water-acetic acid $(25: 30: 45: 0.1)$ adjusted to $\mathrm{pH}$ 5.5 with ammonium hydroxide. The flow-rate was $0.9 \mathrm{ml} / \mathrm{min}$, oven temperature $37^{\circ} \mathrm{C}$.

Explanation of figures: standards $(\bullet) C, D, E$ and $B$ : $t_{R}$ of synthetic LTC $_{4}, \mathrm{D}_{4}, \mathrm{E}_{4}$ and $\mathrm{B}_{4}$ respectively; standards (•)

15-H, 12-H and 5-H: 15-HETE, 12-HETE and 5-HETE, respectively

Identification of peaks (as indicated in Results): $1=$ front $2,3=$ tri-HETEs and lipoxins, $4=\mathrm{LTD}_{4}, 5=\mathrm{LTB}_{4}$

(5-(S),12-(R)-dihydroxy-6,8,10,14-eicosatetraenoic acid), $6=5,12-$ and 8,15-diHETE, $7=$ unknown HETE, $8=15$-HETE, $9=8$ - and/or 11-HETE.

From time 0 absorption was monitored at $280 \mathrm{~nm}$, at time 13 at $270 \mathrm{~nm}$ and at $25 \mathrm{~min}$ at $234 \mathrm{~nm}$. Fractions of 1 per min were collected from 0-18 min for RIA determinations of $\mathrm{LTC}_{4}$ (\$), from 0-23 min for immunoassay of $\mathrm{LTB}_{4}($ (目), from 20-60 min for immunoassay of 12-HETE (ש) and from 20-53 min for the assay of 15-HETE (ख) . Explanation of peaks $a-m$ are given in the Results section.

In fractions 4-8 PGs were measured (data shown in the Table)

as anti-12-HETE, anti-15-HETE and anti-6-ketoPGF $_{1 \alpha}$ antibodies. Standards of 6-keto-PGF ${ }_{1 \alpha}, \mathrm{TxB}_{2}$ and $\mathrm{PGE}_{2}$ were purchased from Sigma (USA), and 
anti-TxB 2 and anti-PGE 2 from l'Institut Pasteur (Paris, France). Anti-LTB ${ }_{4}$ was obtained from Wellcome Research (UK) and a LTC $_{4}$ RIA kit from Dupont de Nemours (NEN Division, Germany).

The cross reactivities $(>0.1 \%)$ at $50 \% \mathrm{~B} / \mathrm{B}_{0}$ were as follows: anti-6. $\mathrm{kPGF}_{1 \alpha}$ : $\mathrm{PGF}_{1 \alpha} 7.8 \%, 6 \mathrm{kPGE}_{1}$ 6.8\%, $\mathrm{PGF}_{2 \alpha} 2.2 \%, \mathrm{PGE}_{2} 0.6 \%$; anti- $\mathrm{TxB}_{2}$ : all $<0.1 \%$; anti-PGE 2 : $\mathrm{kPGE}_{2} 13.2 \%, \mathrm{PGE}_{1} 10.7 \%$; anti-15-HETE: $8,15-$ and 5,15-diHETE 1.0\%, 12HETE 0.5\%; anti-12-HETE: 15-HETE 0.3\%,

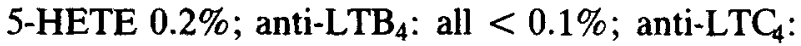
11-tr-LTD $40 \%, \mathrm{LTD}_{4} 55 \%, \mathrm{LTD}_{4}$-sulfone $10 \%$, $\mathrm{LTC}_{4}$-sulfone and $\mathrm{LTE}_{4} 9 \%$. All tritiated compounds were obtained from the Radiochemical Centre (Amersham, UK).

\section{RESULTS}

The Figure shows a separation of lipoxygenase products by HPLC (day 2, not corrected for recoveries). In this chromatogram the absorption at different wave-lengths was monitored (peaks 1-9). Closed dots represent retention times $\left(t_{R}\right)$ of synthetic leukotrienes and mono-HETEs. The detection level was 1 and $5 \mathrm{ng}$ respectively.

After the front two high peaks were measured, indicated by 2 and 3 . Immunoassay of these peaks revealed very low activities to the $\mathrm{LTC}_{4}$ antibody (approx. $0.065 \mathrm{ng}$ max.), as shown in the peaks $b$ and $c$. These compounds could be tri-HETEs or 11trans-LT metabolites. As such derivatives have high cross reactivities to the $\mathrm{LTC}_{4}$ antibody, this is unlikely. Therefore we assume these peaks to be lipoxins (17). Furthermore peaks 4 and d could represent either substances of related structure or 11-trans-LTD . $^{2}$

Peak 5 coelutes with $\mathrm{LTB}_{4}$. The corresponding peak $f$ contains a fraction with a high immunoreactivity to $\mathrm{LTB}_{4}$ (approx. $2.7 \mathrm{ng}$ max.). In the fractions that were eluted after $20 \mathrm{~min}$, the immunoreactivities to 15- and 12-HETE were determined. Both the absorption of peak 7 and the immunoreactivities represented in peaks $g$ and $k$ respectively suggest that it has the structure of a mono-HETE.

Peak 8 coeluted with 15-HETE and showed immunoreactivity to this compound represented in peak 1 . The amount was maximal, approximately $80 \mathrm{ng}$. There is also cross reactivity with the 12HETE antibody.

Although no peak in the chromatogram has the $t_{R}$ of the 12-HETE, two immunoreactive ones were found. These contained small amounts of this substance $(<0.5 \mathrm{ng})$. The compound representing peak 9 is unknown. Peak $j$ finally indicates the presence of very small amounts of 5-HETE.

Two additional methods were used for the identification of peak 8. The absorption spectrum was measured and shown to be identical to that of synthetic 15-HETE. Secondly, the biological activity of the peak was measured on human small airway smooth muscle (18). It was comparable with the small contractile activity of the standard of 15-HETE and more potent than prostaglandins $F_{2 \alpha}$ and $E_{2}$.

The calculated amounts determined by radioimmunoassay were of the same order of magnitude as those obtained by measurement of the absorption spectrum at the wavelengths indicated. In the Table the mean concentrations of eicosanoids in mucus obtained from morning stools during 4 days are given. Amounts are corrected for recoveries (15) and expressed in $\mathrm{ng} / \mathrm{g}$ mucus.

Table Concentrations of eicosanoids in mucus of acute ulcerative proctocolitis obtained from morning stool during. 4 days (amounts in $\mathrm{ng} / \mathrm{g}$ mucus).

\begin{tabular}{cc}
\hline 6-keto-PGF & $3.7 \pm 1.4$ \\
TXB $_{1 \alpha}$ & $9.2 \pm 2.5$ \\
PGE $_{2}$ & $2.0 \pm 0.4$ \\
LTB $_{4}$ & $21 \pm 3.0$ \\
LTC $_{4}$ & $\leq 0.8$ \\
$15-H E T E$ & $530 \pm 65$ \\
\hline
\end{tabular}

\section{DISCUSSION}

The results indicate that the amounts of $\mathrm{LTB}_{4}$ and in particular 15-HETE found in the rectal mucus in ulcerative procto colitis are considerably higher than the amounts of PGs. Furthermore smaller amounts are formed of two other mono-HETEs and a di-HETE. Comparatively small, but detectable, amounts of sulphidopeptide LTs were measured by RIA. Two other non treated patients with ulcerative proctocolitis were studied once. Amounts of $\mathrm{LTB}_{4}$ and 15-HETE were in the same range as those shown is this report. Since it is not possible to obtain mucus from normal individuals other than by biopsies the results reported here only support the possible important role of mono-hydroxy acids in active ulcerative colitis. Measurements of exogenous 15-HETE, $\mathrm{LTB}_{4}$ and $\mathrm{PGE}_{2}$ in controls and patients with ulcerative colitis generated from ${ }^{14} \mathrm{C}$ arachidonic acid labelled biopsies confirmed the ratios found in the herewith presented results (unpublished results).

Recent studies of experimental colitis have established a relationship between $\mathrm{LTB}_{4}$ and $\mathrm{LTC}_{4}$ and the severity of the inflammation. Eicosanoid production progressively increased during development of inflammation and infiltration of inflammatory cells (9). In the model used methylprednisolone was not a potent suppressor of LT production (19). In Crohn's disease, enhanced $\mathrm{LTB}_{4}, \mathrm{C}_{4}$ and $\mathrm{D}_{4}$ production by colonic mucosa has been described. This formation was dose-dependently inhibited by sulphasalazine and 5-aminosalicylic acid (11). The 
sulfidopeptide $\mathrm{LTs} \mathrm{C}_{4}, \mathrm{D}_{4}$ and $\mathrm{E}_{4}$ are known to increase mucus production (13), vascular permeability (20) and to contract intestinal smooth muscle (21). The chemotactic $\mathrm{LTB}_{4}$ has also been shown to increase vascular permeability (22), to contract smooth muscle and to increase epithelial secretion (23).

In small bowel mucosa from celiac patients it was found that 15-HETE formation was increased after in vitro challenge with gluten (24). Mono-HETEs have been shown to act as mediators of tracheal mucus secretion $(13,25)$, and have inflammatory effects in the skin (14). Additionally it was found that 15-HETE inhibits the formation and the chemotactic response of neutrophils to $\mathrm{LTB}_{4}$, determined in synovial fluid in experimental arthritis (26). This may lead to an increase of cyclooxygenase products. In turn misoprostol, a $\mathrm{PGE}_{1}$ analogue, can cause an inhibition of the initiating event and the amplification of the inflammation caused by $\mathrm{LTB}_{4}(27)$.

In our study there was a linear relationship between the 15-HETE and PG contents, whereas the amount of $\mathrm{LTB}_{4}$ was inversely proportional to that of the PG (data not shown).

The implications of our observation, that 15HETE is present in high concentrations in mucus, for the treatment of ulcerative colitis, are not clear. Though 5-ASA is a rather weak inhibitor of 5lipoxygenase the use of more active and specific 5 -lipoxygenase inhibitors in ulcerative colitis is being considered (28). Selective 5-lipoxygenase inhibitors, however, could result in increased 15-HETE, diHETE and lipoxin production, and may therefore prove disappointing anti-inflammatory agents for inflammatory bowel disease.

\section{Acknowledgements}

The authors are indebted to Drs. J. E. Vincent and M. van Blankenstein for evaluation of the manuscript.

\section{References}

1. Donowitz M. Arachidonic acid metabolites and their role in inflammatory bowel disease. Gastroenterology 88: 580-587, 1985.

2. Sharon P, Stenson W F. Metabolism of arachidonic acid in acetic acid colitis in rats. Gastroenterology 88: $55-63,1985$

3. Sharon P, Stenson W F. Enhanced synthesis of leukotriene $\mathrm{B}_{4}$ by colonic mucosa in inflammatory bowel disease. Gastroenterology 86: 453-460, 1984.

4. Lauritsen $K$, Laursen L S, Bukhave $K$, et al. Effects of topical 5-aminosalicylic acid and prednisolone on prostaglandin $\mathrm{E}_{2}$ and leukotriene $\mathrm{B}_{4}$ levels determined by equilibrium in vivo dialysis of rectum in relapsing ulcerative colitis. Gastroenterology 91: 837-844, 1986

5. Lauritsen $K$, Laursen L S, Bukhave $K$, et al. In vivo proviles of eicosanoids in ulcerative colitis, Crohn's colitis and clostridium difficile colitis. Gastroenterology 95: 11-17, 1988.

6. Tornhamre S, Edenius C, Smedegård G, Sjöquist $B$ and Lindgren J A. Effects of sulfasalazine and a sulfasalazine analoque on the formation of lipoxygenase and cyclooxygenase products. Eur. J Pharmacol. 169: 225-234, 1989.

7. Wallace J L. 5-Lipoxygenase: a rational target for therapy of infiammatory bowel disease? TiPS 11: $51-53,1990$.

8. Wallace J L, MacNaughton W K, Morris G P and Beck P L. Inhibition of leukotriene synthesis markedly accelerates healing in a rat model of inflammatory bowel disease. Gastroenterology 96: 29-36, 1989.

9. Zipser R D, Nast C C, Lee $M, K a o H ~ W$ and Duke $R$. In vivo production of leukotriene $B_{4}$ in rabbit colitis. Gastroenterology 92: 33-39, 1987.

10. Okayasu I, Hatakeyama $S$, Yamada $M$, Ohkusa $T$, Inagaka $Y$ and Nakaya $R$. A novel method in the induction of reliable experimental acute and chronic ulcerative colitis in mice. Gastroenterology 98: 694-702, 1990 .

11. Peskar B M, Dreyling K W, Peskar B A, May B and Goebell $H$. Enhanced formation of sulfidopeptide-leukotrienes in ulcerative colitis and Crohn's disease; inhibition by sulfasalazine and 5-aminosalicylic acid. Agents and Actions 18: 381-383, 1986

12. Nielson $\mathrm{O} \mathbf{H}$, Thomson $\mathbf{M} K$, Kissmeyer $\mathbf{A} \mathbf{M}$, Lanholz E, Kirstein D and Ahnfelt-Rönne I. Effect of the leukotriene $D_{4} / E_{4}$ antagonist, SR2640, in chronic inflammatory bowel disease: an open clinical study. Proc. 7th Intern. Confer. Prostagl. Florence, 221, 1990.

13. Johnson $H \mathrm{G}$, McNee $M L$ and Sun F F. 15-Hydroxyeicosatetraenoic acid is a potent infiammatory mediator and agonist of canine tracheal mucus secretion. Am. Rev. Respir. Dis. $131917-922,1985$.

14. Higgs G A, Salmon J A, Spayne J A. The inflammatory effects of hydroperoxy and hydroxy acid products of arachidonate lipoxygenase in rabbit skin. Br. J. Pharmac. 74: 429-433, 1981.

15. Zijlstra F J. Disabilities and how to live with them: ulcerative colitis. Lancet i: 215-216, 1982.

16: Zijlstra F J, Vincent J E. Determination of leukotrienes and prostaglandins in ${ }^{14} \mathrm{C}$-arachidonic acid labelled human lung tissue by high-performance liquid chromatography and radioimmunoassay. J. Chromatogr. 311: 39-50, 1984.

17. Walstra $P$, Verhagen $J$, Vermeer $M$, et al. Evidence for lipoxin formation by bovine polymorphonuclear leukocytes via triple dioxygenation of arachidonic acid. FEBS lett. 228: 167-171, 1988.

18. Zijlstra F J, Naaktgeboren $M$, Mons $H$ and Vincent $J \mathrm{E}$. Formation of prostaglandins and leukotrienes by human lung tissue in vitro after activation by the calcium ionophore A23187. Eur. J. Clin Invest. 17: 325-329, 1987.

19. Zipser R D, Kao H W, Nast C C. In vivo production of leukotrienes in experimental colitis. Adv. Prostagl. Leukotr. Res. 17: 344-346, 1987.

20. Soter N A, Lewis R A, Corey E J and Austen $\mathrm{K} F$. Local effects of synthetic leukotrienes $\left(\mathrm{LTC}_{4}\right.$, $\mathrm{LTD}_{4}, \mathrm{LTE}_{4}$ and $\mathrm{LTB}_{4}$ ) in human skin. J. Invest. Dermatol. 80: 115-119, 1983.

21. Pawlik W W, Gustav P, Sendur R, Czarnobilski K, Konturek S J, Beck $G$ and Jendralla $M$. Vasoactive and metabolic effects of leukotriene $C_{4}$ and $D_{4}$ in the intestine. Hepatogastroenterol 35: 87-90, 1988.

22. Jackson $R \mathbf{M}$, Ann $H$ S. Effects of leukotriene $B$ on permeability, prostacyclin and thromboxane release by normal and oxygen-preexposed isolated, perfused rat lungs. Prostagl. Leukotr. EFA 32: $121-128,1988$.

23. Goetzl E J, Burall B A, Baud L, Scriven K H, Levine $J \mathrm{D}$ and Koo $\mathrm{CH}$. Generation and recognition of leukotriene mediators of hypersensitivity and inflammation. Digest. Dis. Sci 33: $36 \mathrm{~S}-40 \mathrm{~S}, 1988$. 
24. Krilis A K, MacPherson J L, de Carle D J, Daggard G E, Tally N A and Chesterman C N. Small bowel mucosa from celiac patients generates 15-hydroxyeicosatetraenoic acid (15-HETE) after in vitro challenge with gluten. J. Immunol. 137: 3768-3771, 1986.

25. Marom Z, Shelhamer J H, Sun F and Bach M K. Human airway monohydroxy eicusatetraenoic acid generation and mucus release. J. Clin. Invest. 72: 122-127, 1983.

26. Fogh K, Hansen E S, Herlin T, Knudsen V, Henriksen T B, Ewald $H$, Bunger $C$ and Kragballe
K. 15-Hydroxyeicosatetraenoic acid (15-HETE) inhibits carragheenan-induced experimental arthritis and reduces synovial fluid leukotriene $B_{4}$ (LTB $)$. Prostaglandins 37: 213-227, 1989.

27. Fedorak R N, Empey L R, ManArthur C and Jewell L D. Misoprostol provides a colonic mucosal protective effect during acetic acid-induced colitis in rats. Gastroenterology 98: 615-625, 1990.

28. Laursen L S, Naesdal J, Bukhave K, Lauritsen K and Rask-Madsen J. Selective 5-lipoxygenase inhibition in ulcerative colitis. Lancet 335: 683-685, 1990 